\title{
EFECTOS SECUNDARIOS DE LA TRIAMCINOLONA EN PACIENTES JÓVENES
}

\section{SIDE-EFFECTS OF TRIAMCINOLONE IN YOUNG PATIENTS}

\author{
SUÁREZ-FIGUEROA M ${ }^{1}$, CONTRERAS I ${ }^{2}$, NOVAL $\mathrm{S}^{2}$
}

\begin{abstract}
RESUMEN
Casos clínicos: El uso de triamcinolona intravítrea está extendiéndose como tratamiento de enfermedades maculares. Los efectos secundarios pueden variar en jóvenes. Presentamos dos casos de incremento de la presión intraocular (PIO) y desarrollo de cataratas. Dos pacientes jóvenes con uveítis crónicas refractarias a glucocorticoides perioculares y orales, recibieron inyecciones intravítreas de triamcinolona.

El edema macular crónico mejoró en ambos pacientes. Se produjo un incremento de PIO, seguido de la aparición acelerada de cataratas.

Discusión: La juventud y la inflamación crónica podrían estar asociadas a incrementos de PIO y el consecuente desarrollo de cataratas tras inyección intravítrea de triamcinolona.

Palabras claves: Juventud, triamcinolona intravítrea, efectos secundarios, uveítis, catarata, edema macular.
\end{abstract}

\begin{abstract}
Case reports: Intravitreal triamcinolone is being increasingly employed for the treatment of macular diseases. We report two cases of intraocular pressure elevation and cataract formation after intravitreal triamcinolone therapy, and wonder if these complications are more likely when this agent is used in young patients.

Intravitreal triamcinolone was injected into both eyes of the two young patients with chronic posterior and intermediate uveitis refractory to peribulbar and oral corticosteroid therapy. Chronic cystoid macular edema improved in both patients, however the intraocular pressure increased, requiring topical antihypertensive therapy, and this was followed by accelerated cataract formation.

Discussion: Young age and chronic inflammation could be associated with an intraocular pressure rise and subsequent cataract development after intravitreal triamcinolone (Arch Soc Esp Oftalmol 2006; 81: 405-408).
\end{abstract}

Key words: Youth, intravitreal triamcionolone, side effects, uveitis, cataract, macular edema.

Recibido: 12/12/05. Aceptado: 19/7/06.

Hospital Ramón y Cajal. Madrid. España.

1 Doctora en Medicina.

2 Licenciada en Medicina.

Ninguno de los autores tienen interés comercial en ninguno de los productos mencionados en este trabajo. Tampoco hemos recibido apoyo económico para la realización de este trabajo.

Correspondencia:

Inés Contreras

C/. Martín de los Heros, $80,5 .^{\circ} \mathrm{D}$

28008 Madrid

España

E-mail: inescon3@yahoo.com 


\section{INTRODUCCIÓN}

La inyección de triamcinolona intravítrea está cada vez más extendida como tratamiento de las enfermedades maculares de origen inflamatorio y neovascular. Sin embargo, hay poca información disponible sobre su uso en pacientes jóvenes. Presentamos dos casos de incremento de la presión intraocular seguidos de la aparición de cataratas tras la inyección de triamcinolona intravítrea en dos pacientes con uveítis crónica.

\section{CASOS CLÍNICOS}

\section{Caso 1}

Niña de 13 años de edad, diagnosticada en mayo de 2003 de uveítis intermedia y tratada con glucocorticoides tópicos, peribulbares y orales, con edema macular quístico (EMQ) persistente en ambos ojos y agudeza visual (AV) de 0,2 en ojo derecho (OD) y 0,4 en el ojo izquierdo (OI). En febrero de 2004 se inyectaron $4 \mathrm{mg}$ de triamcinolona intravítrea (Trigon Depot ${ }^{\circledR}$, Bristol-Myers Squibb) en el OD. Un mes después, el EMQ se había resuelto con $\mathrm{AV}$ de 0,7 , por lo que se repitió el tratamiento en el OI con AV de 0,7. Un año después, se detectaron presión intraocular (PIO) de $26 \mathrm{mmHg}$ OD y 23 $\mathrm{mmHg}$ OI que fueron controladas con timolol 0,5\% tópico (Timoftol ${ }^{\circledR} 0,5 \%$, MSD). En julio de 2005, había desarrollado cataratas subcapsulares posteriores (fig. 1), con AV de 0,2 OD y 0,5 OI. Actualmente está pendiente de cirugía de facoextracción.

\section{Caso 2}

Niña de 13 años de edad que consultó por pérdida de $\mathrm{AV}$ en ambos ojos (0,1 OD y 0,2 OI ). En el examen de fondo de ojo se objetivó la presencia de EMQ bilateral. En la angiografía fluoresceínica presentaba una epiteliopatía pigmentaria difusa con edema exudativo y desprendimientos retinianos serosos secundarios. A los 5 meses de edad, había sido tratada con éxito de una histiocitosis de células de Langerhans, por lo que se realizó un estudio sistémico completo, incluidas pruebas de imagen craneal y orbitarias. No se encontró ningún hallazgo significativo. Se inició terapia con corticosteroides tópicos, peribulbares y orales.

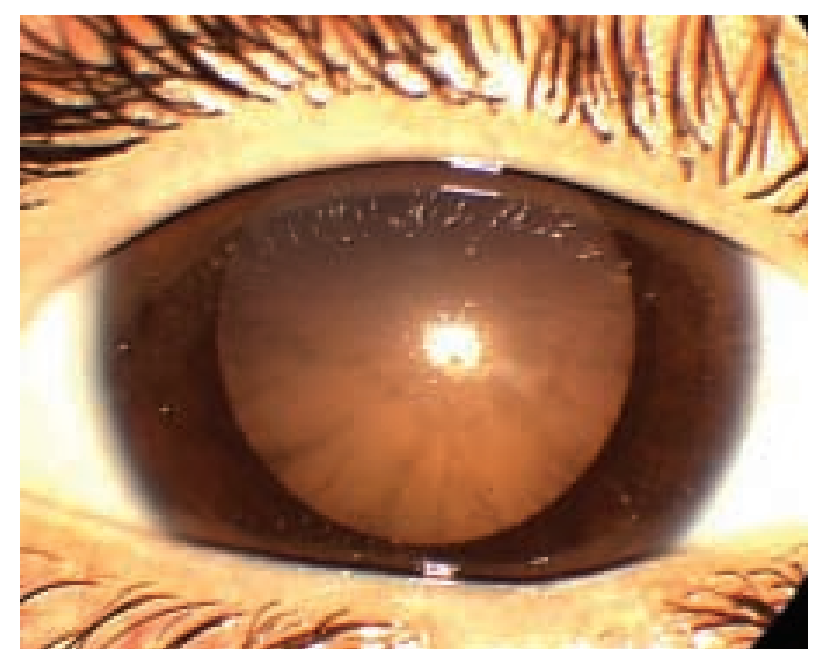

Fig. 1: Caso 1. Desarrollo de catarata subcapsular posterior tras triamcinolona intravítrea y aumento de la presión intraocular.

Las inyecciones de triamcinolona peribulbar se repitieron en cuatro ocasiones a lo largo del siguiente año, logrando sólo mejorías transitorias del edema macular y los desprendimientos serosos. En septiembre de 2002, comenzó tratamiento con ciclosporina oral y en marzo de 2003 se añadió azatioprina, debido a la persistencia del EMQ. Finalmente, se inyectaron $4 \mathrm{mg}$ de triamcinolona intravítrea en ambos ojos en julio de 2003 con mejoría inicial del EMQ y de la AV. Se repitieron las inyecciones dos veces en el OD y una vez más en el OI. En diciembre de 2004, se registraron PIO de $27 \mathrm{mmHg}$ OD y $26 \mathrm{mmHg}$ OI, por lo que se indica terapia tópica con timolol $0,5 \%$ y dorzolamida $0,02 \%$ $\left(\right.$ Cosopt $\left.^{\circledR}\right)$. En marzo de 2005, la paciente presenta pérdida de AV severa bilateral $(<0,05)$ por desarrollo de cataratas intumescentes (fig. 2). Tras cirugía de facoextracción con implantación de lente intraocular sin complicaciones, la $\mathrm{AV}$ es de 0,3 en ambos ojos.

\section{DISCUSIÓN}

El incremento de la PIO es una complicación conocida de las inyecciones intravítreas de triamcinolona, con una incidencia del $30 \%$ aproximadamente. Jonas et al han demostrado que la aparición de hipertensión intraocular tras triamcinolona intravítrea está asociada significativamente a una menor edad y a uveítis $(1,2)$. 


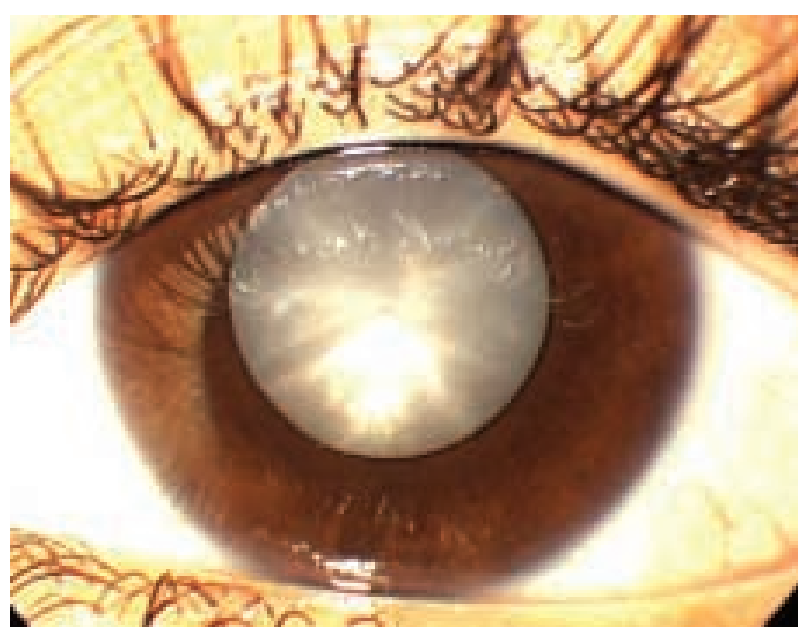

Fig. 2: Caso 2. Desarrollo de catarata intumescente tras triamcinolona intravitrea e incremento de presión intraocular.

Young et al, en un estudio piloto prospectivo con triamcinolona intravítrea para EMQ crónico idiopático inflamatorio encontraron un aumento de PIO en 5 de 6 pacientes, con edades comprendidas entre 23 y 57 años. Especularon que esta alta incidencia podría deberse a un compromiso en el drenaje angular secundario a la inflamación crónica (3).

En el único caso que hemos encontrado publicado de triamcinolona intravítrea en jóvenes, el paciente de 17 años desarrolló hipertensión ocular (4).

Nuestras dos pacientes presentaron incrementos de PIO, lo que apoya los hallazgos de Jonas et al por los que la menor edad y la inflamación propician los picos hipertensivos tras triamcinolona intravítrea.

La rápida aparición de cataratas en ambas pacientes podría estar relacionada con el uso prolongado de glucocorticoides; sin embargo, Gillies et al han encontrado relación entre los aumentos de
PIO tras triamcinolona y el desarrollo de catarata. En un estudio prospectivo de degeneración macular asociada a la edad exudativa tratada con triamcinolona intravítrea, detectaron una tasa significtivamente mayor de progresión de cataratas subcapsular posterior y cortical en pacientes con respuestas hipertensivas respectos a los no respondedores ( $51 \%$ vs $3 \%$ y $15 \%$ vs $3 \%$ respectivamente) (5). En el estudio de Young et al, 2 de 5 respondedores desarrollaron catarata subcapsulares posteriores en 6 meses (3). Por lo tanto, la aparición acelerada de cataratas en nuestras pacientes podría estar relacionada con los incrementos de PIO.

Los oftalmólogos que se planteen el uso de triamcinolona en jóvenes, deberían tener presente el mayor riesgo de hipertensión ocular y de la formación de cataratas. Es necesaria más información sobre los efectos secundarios de la triamcinolona en estos pacientes y sobre cómo la etiología inflamatoria influye en la respuesta de la presión intraocular.

\section{BIBLIOGRAFÍA}

1. Jonas JB, Kreissig I, Degenring R. Intraocular pressure after intravitreal injection of triamcinolone acetonide. $\mathrm{Br}$ J Ophthalmol 2003; 87: 24-27.

2. Jonas JB, Degenring RF, Kreissig I, Akkoyun I, Kamppeter BA. Intraocular pressure elevation after intravitreal triamcinolone acetonide injection. Ophthalmology 2005; 112: 593-598

3. Young S, Larkin G, Branley M, Lightman S. Safety and efficacy of intravitreal triamcinolone for cystoid macular oedema in uveitis. Clin Experiment Ophthalmol 2001; 29: 2-6.

4. Degenring RF, Jonas JB. Intravitreal injection of triamcinolone acetonide as treatment for chronic uveitis. $\mathrm{Br} J$ Ophthalmol 2003; 87: 361.

5. Gillies MC, Kuzniarz M, Craig J, Ball M, Luo W, Simpson $J M$. Intravitreal triamcinolone-induced elevated intraocular pressure is associated with the development of posterior subcapsular cataract. Ophthalmology 2005; 112. 139-143. 